\title{
LA CERTEZA DE LOS CRITERIOS
}

\author{
Ricardo A. Guibourg \\ Universidad de Buenos Aires
}

\section{Especies criteriosas}

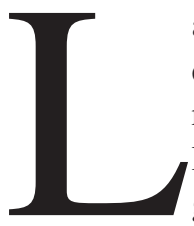

a ameba extiende un seudópodo en busca de alimento. Si lo que encuentra la satisface, lo incorpora. En caso contrario, lo rechaza y retrae su seudópodo para probar en otra dirección. El organismo unicelular tiene y usa algún criterio para distinguir lo que le conviene de lo que prefiere desechar. No importa ahora cuáles sean el soporte, la naturaleza ni el grado de conciencia o de autonomía de esos criterios. Tampoco si ellos son acertados o si la ameba se equivoca a veces. Quiero destacar, en cambio, que los organismos vivos -y el hombre entre ellos- dependemos de nuestra capacidad para desarrollar métodos, primitivos y genéticos o culturalmente elaborados, con los que distinguimos unos acontecimientos de otros a partir de nuestros intereses. Tal es el modo en que, de hecho, nos relacionamos con el mundo, tratamos de comprenderlo y logramos, de tanto en tanto, obtener de él lo que deseamos o escapar de alguno de sus peligros.

El criterio de la ameba, que he usado como ejemplo, es muy sencillo: apenas un mecanismo de reacción ante estímulos probablemente químicos. El ser humano tiene reacciones parecidas ante un estruendo repentino, o al sentir una quemadura, o frente a una agresión física. Pero sobre esa base, y con la ayuda de su inteligencia y del lenguaje, ha llegado a establecer sistemas muy complejos de criterios que se entrelazan en una tupida red y se extienden por diversos niveles de abstracción. El solo hecho de distinguir unos objetos de otros muestra el uso de criterios (acaso fundados en la forma, el color o el movimiento) para recortarlos mentalmente de la realidad. El lenguaje exige criterios de clasificación en los que se escogen como definitorias unas características en lugar de otras. Los enunciados descriptivos, fuera de hallarse formalmente sujetos a criterios de aceptabilidad sintáctica y semántica, no son todos equivalentes: algunos dan buen resultado cuando se 
los constituye en guías de nuestras creencias y actitudes (por ejemplo, creer que llueve y llevar paraguas), en tanto otros conducen a consecuencias no deseadas (creer que hay buen tiempo y llegar a destino empapados). Tenemos criterios para decidir qué creer: en este caso, interpretar en cierta forma lo que vemos por la ventana. Pero no todas las descripciones se refieren a hechos actuales y fácilmente verificables, de modo que desarrollamos criterios, bastante inseguros, para decidir a quién hemos de creer cuando relata un acontecimiento o qué fenómenos conocidos o creídos pueden juzgarse indicios de lo que ocurrirá en el futuro.

Los enunciados de hecho se hallan indirectamente relacionados con nuestros intereses, apetencias o temores, porque el mejor servicio de esas pasiones es, al fin de cuentas, el último criterio con el que juzgamos la aceptabilidad del marco teórico en el que construimos y discernimos las descripciones. Pero, paralelamente a la elaboración de los enunciados y a menudo interfiriendo con ellos, nuestras preferencias generan criterios diferentes, también en distintos niveles. Es claro que deseamos algunos acontecimientos y tememos o aborrecemos otros: ese discernimiento básico se manifiesta en la preferencia por ciertas conductas propias o ajenas que, sin ser necesariamente en sí mismas el objeto de nuestro deseo o de nuestro aborrecimiento, supuestamente facilitan o tienden a impedir la aparición de aquellos fenómenos. En esta línea también hay varios niveles de abstracción, pero en cada uno de esos niveles inciden, además, las predicciones, correctas o equivocadas, que hacemos acerca de la probabilidad de acontecimientos futuros y de la relación causal que nos permita preverlos, provocarlos o evitarlos desde el presente. Y, a su vez, las predicciones se ven influidas por nuestro wishful thinking, así como las palabras que empleamos en las descripciones de hecho se hallan teñidas por el contenido emotivo que les asignamos.

El cruce de unos criterios con otros, a menudo pertenecientes a niveles distintos, ha contribuido a generar perplejidades que todavía luchamos por resolver. El debate de los universales, el fundamento de las ciencias formales, la aporía de la inducción, la teoría del lenguaje, el discurso moral y sus presupuestos metaéticos, la construcción del fenómeno jurídico y las controversias acerca de su descripción, la interpretación de la ley y su relación con la política y con la moral, las reglas con las que los jueces deciden las causas y el modo en que los juristas describen y critican esas decisiones son ejemplos muy diversos entre sí de controversias acerca del uso de criterios, cuando no se dispone de metacriterios decidibles para resolverlas.

Por encima de su diversidad, todas esas controversias tienen, pues, algo en común. Ellas persiguen, como resultado final, la construcción, adquisición o determinación de criterios, esto es, disposiciones para preferir un es- 
tado de cosas a otro o para usar un enunciado, en lugar de su opuesto, como base para constituir nuestras creencias o apoyar en ellas nuestras actitudes.

\section{Grupos y niveles de criterios}

Los criterios corresponden a distintas apetencias (gastronomía, escultura, curiosidad, abrigo, esparcimiento, seguridad, descanso, salud y otras semejantes), así como a la elaboración de conceptos e instrumentos tendientes a generalizarlas, definirlas o facilitarlas (nouvelle cuisine, técnica escultórica, metodología, arquitectura, corte y confección, moral, derecho, medicina) y aun a regir la construcción de esos mismos conceptos e instrumentos (estética, ética, epistemología, matemática, lógica) o, en última instancia, a trazar los parámetros básicos del pensamiento (filosofía). El hombre ha agrupado los criterios por el tema al que se refieren, por el método que contribuye a su adopción, por su nivel de abstracción o empleando a la vez dos o más de esos metacriterios clasificatorios. Tales grupos reciben nombres como biología, derecho, fisiología, informática, aviación, medicina, matemática, moral, ética, astrología, mecánica, amistad.

Una vez aproximadamente delimitados, los agrupamientos de criterios suelen imaginarse como objetos reales, dotados de características propias, y empieza a debatirse cuál sea la confiabilidad genérica de cada grupo o cuáles las relaciones entre ellos, relaciones que no pocas veces se postulan o se desestiman también como hechos verificables.

Las reflexiones acerca del derecho exhiben varias de esas perplejidades. Es notorio que exigimos a los sistemas normativos la satisfacción de una estructura deductiva consistente y preferiblemente completa, pero no nos damos por conformes si ella no da cuenta aproximada del ejercicio real del poder, mientras una y otra vez se busca inyectar en la descripción del derecho algún tipo de contenidos a los que somos particularmente afectos, llámense propiedad, familia, democracia, derechos humanos o liberación de los pueblos. Una teoría unificada es impracticable; la combinación de varias teorías carecería de compatibilidad metodológica; pero, aun en esas circunstancias, los juristas siguen girando en torno a sus apetencias frustradas y buscan siempre el modo de dar cuenta de aquellas relaciones.

No pretendo ofrecer una solución al problema; pero creo posible ensayar un metacriterio ordenador de los grupos de criterios que, acaso, ayudaría a reexaminar los vínculos entre grupos y, a la vez, a explicar y tal vez disolver algunas perplejidades.

En los criterios que empleamos, cualesquiera sean, es posible observar una tensión entre los puntos de vista individual y colectivo. El individuo acepta, en principio, los criterios que le convienen. Pero, cuando considera 
útil lograr que otros los compartan, se ve obligado a hacer concesiones tendientes a facilitar la aceptación colectiva. Una vez modificados y relativamente fijados por su intersubjetividad, los criterios ya no son tan apropiados para su aceptación por cada individuo, a menos que su generalización ofrezca beneficios adicionales específicos. A mayor aceptabilidad colectiva, pues, corresponde en principio menor garantía de aceptación individual.

Si se parte de esta relación entre grupos de criterios, se hace posible ordenarlos en sentido creciente de aceptabilidad colectiva, aun con el riesgo de menor aceptación individual que acabo de mencionar. Destaco, por cierto, que el orden establecido no prejuzga acerca de su precedencia histórica ni lógica.

Nivel 1: Preferencia. El primer peldaño es la simple preferencia. Cada uno prefiere lo que en cada momento cree que le conviene, sin sujeción alguna a reglas ni a reclamos de coherencia entre dos o más opciones sucesivas.

Nivel 2: Principios y valores. Cuando es preciso compatibilizar las preferencias de un sujeto con las de los demás, los acuerdos (no siempre pacíficamente alcanzados) adquieren el nombre de principios o, en un nivel más abstracto y general, el de valores. Los estados de cosas son valorados por aplicación de ciertos criterios comunes y las preferencias individuales no son tenidas en cuenta cuando no guardan coherencia con las valoraciones establecidas.

Nivel 3: Moral. Los valores -como abstracción y generalización de las valoraciones acordadas a partir de una transacción entre preferencias individuales- no son por sí solos suficientes para extrapolarse hacia decisiones prácticas. La identificación de los valores no es universalmente acordada, su interpretación y su alcance quedan sujetos a modalidades individuales de aplicación, los habituales casos de conflicto entre ellos se zanjan mediante metapreferencias también individuales que conducen a soluciones opuestas. Y, lo que es más grave, las personas aprecian la aplicabilidad de valores y principios desde su propio punto de observación, que introduce la variable de la cercanía del observador respecto de cada problema y respecto de cada circunstancia en que los principios puedan verse involucrados en un mismo problema.

Se hace preciso, pues, poner los principios en acción de un modo que también sea intersubjetivamente aceptable. Para intentar el cumplimiento de este objetivo se elaboran reglas morales. En ellas se prescriben directamente las conductas, a fin de que, una vez aceptado el sistema normativo moral, ningún individuo pueda invocar un modo diverso y personal de aplicación de los principios y valores en los que dicho sistema se funde. 
Nivel 4: Derecho. La moral -cualquiera sea el contenido que se le asigne- es más intersubjetiva que la mera preferencia individual; pero, consecuentemente, importa ya un serio riesgo de conflicto con esa preferencia. Cada comunidad intenta reducir ese riesgo mediante la educación. Ella tiende, entre otras cosas, a lograr la internalización de las normas morales en la conciencia individual; esto es, a construir mecanismos psicológicos que aseguren que, en caso de conflicto entre la norma y la preferencia (ahora llamada tentación), la norma salga triunfante y la preferencia sea reprimida y sustituida por el respeto de la norma.

La prédica moral, sin embargo, no es suficiente para garantizar suficientemente el resultado apetecido. Muchos individuos se sienten internamente libres para rechazar la moral aprendida o parte de ella, e incluso se forman agrupamientos de personas dotadas de rebeldías con contenido aproximadamente semejante, en las que cada uno encuentra un sustituto de la contención y de la pertenencia que el sistema moral dominante pudiera negarle. Pero aún hay más: incluso dentro del acatamiento de un mismo sistema moral, la identificación de las propias normas es susceptible de controversias, y tales controversias no disponen de un criterio intersubjetivo dotado de consenso que permita resolverlas de manera definitiva y confiable. En definitiva, pues, en cada sociedad coexisten dos o más sistemas morales parcialmente conflictivos entre sí (y a menudo internamente inconsistentes), con el agravante de que cada uno de ellos es identificado por sus propios adherentes con un sesgo incontrolablemente individual.

Cubrir esa falencia es el objetivo del derecho. A partir del sistema moral dominante en cada momento, interpretado por quienes ejerzan el poder efectivo, se traza un sistema normativo parecido pero más minucioso, que introduce varios factores de mayor certeza: criterios públicos para la identificación de sus normas y hasta de su contenido, maneras formales para la creación, modificación y derogación de tales normas, mecanismos también públicos y formales para dirimir las controversias y, por encima de todo, un repertorio de sanciones destinado a asegurar la preeminencia del sistema jurídico por encima de las preferencias individuales, cualquiera sea la interferencia del sistema moral al que el sujeto adhiera.

Nivel 5: Predicción. La introducción del sistema jurídico no resuelve, desde luego, los problemas observados en el sistema moral, ya que ellos se reproducen en su seno por vía de las variaciones interpretativas y de la relativa impredecibilidad de las decisiones judiciales. Sin embargo, obtiene una notable ventaja de orden cuantitativo, en especial porque cada controversia en particular puede resolverse de un modo indubitable aunque sea $e x$ post facto, cualquiera sea el acierto o el error que cada uno atribuya más tarde a la decisión. Al mismo tiempo, la amenaza de sanciones contribuye con 
cierta eficacia, si no a garantizar la conformidad de las conductas con las normas, por lo menos a uniformar cierta actitud externa de acatamiento hacia normas cuyo texto se halla públicamente identificado.

A partir de aquí, el juicio de valor acerca de las conductas pertenece a la moral, la validez del derecho puede definirse como la derivación de cada norma dentro de un sistema cuyas bases se asumen como punto de referencia para la construcción del sistema y la eficacia del derecho se concibe como la eventual conformidad efectiva de las conductas con las normas.

En este punto, importa predecir las conductas, no sólo para determinar la eficacia de las normas sino para determinar nuestras actitudes acerca de ellas y también para prevenir o modificar los hechos futuros. El acatamiento general de las normas jurídicas, cuando sucede, constituye un factor favorable a la predecibilidad de las conductas individuales; pero es apenas uno de los muchos factores que pueden incidir en la decisión de los súbditos.

El tema de la conducta, pues, se integra aquí al problema más general de la predicción de los hechos. El esquema predictivo se halla presidido, en nuestros tiempos, por la interpretación causal de universo, que da lugar a la estructura de las ciencias empíricas. La causalidad, es preciso decirlo, ha dado hasta ahora un excelente resultado. No todo puede predecirse, desde luego, porque ignoramos las mayoría de las condiciones iniciales y apenas hemos establecido cierto número de regularidades a las que llamamos leyes naturales. Sin embargo, en ciertos aspectos ha sido posible aislar los fenómenos de tal modo que fuera posible reproducirlos en forma experimental; y en otros aspectos se han trazado leyes de tendencia, en las que la ciencia reconoce ignorar cierto número de condiciones que determinan los casos individuales pero, a pesar de eso, logra enunciar regularidades estadísticas dotadas de la misma pretensión de permanencia que las leyes empíricas más precisas.

Los criterios de predicción, sujetos a un notable factor de ignorancia, tienen eficacia muy variable para la averiguación del futuro; pero, a pesar de eso, muestran una diferencia cualitativa con los criterios jurídicos o morales: se ven, en principio, menos influidos por las preferencias individuales. En efecto, cualesquiera sean nuestra apetencia o nuestra aprensión por un hecho futuro, tendemos a confiar en los indicios objetivos para creer que acaecerá o no acaecerá. Claro está que nuestros sentimientos pueden turbar nuestro juicio a la hora de conceder relevancia a los llamados indicios objetivos; pero esa turbación, una vez advertida, tiende a ser corregida por el propio observador: cada uno tiene interés en obtener la predicción más probable, precisamente para defender mejor sus otras preferencias. 
Nivel 6: Descripción. La incertidumbre presente en la predicción se reduce drásticamente en la descripción de los hechos actuales o pretéritos. Todavía faltan datos acerca de hechos poco conocidos o de acontecimientos históricos, pero los que están a nuestra disposición ofrecen bases más sólidas para la descripción. Los criterios de aceptabilidad tienen por centro a la observación empírica, frente a la cual otros criterios son unánimemente desplazados. Cuando ella falta, los criterios sustitutos (indicios retrospectivos como en la arqueología, referencias de terceros como en los testimonios judiciales o en las crónicas históricas) se examinan rigurosamente de acuerdo con su confiabilidad, se comparan entre sí y se juzgan, en definitiva, según su mayor o menor cercanía o semejanza con la observación directa. No todos los hechos se conocen, nuestra descripción de los hechos conocidos se halla siempre sujeta a revisión y corrección y, además, el conocimiento imperfecto de los indicios da lugar a conjeturas divergentes. Sin embargo, la aceptabilidad de los criterios descriptivos es mucho mayor que la de los criterios predictivos e incomparablemente mayor que la de los criterios normativos o de preferencia. Su aceptación efectiva también es alta, porque los métodos para la averiguación de la verdad (concepto que tiene su aplicación paradigmática en el ámbito descriptivo) reportan a todos un beneficio mensurable también en términos de preferencia individual: todos queremos saber, en cada caso, cuál es la situación que de hecho nos rodea.

Nivel 7: Deducción. Nuestra actividad cotidiana en la elaboración y aplicación de criterios -y, en especial, en el uso del criterio de verdad típico de la actividad descriptiva- ha dado lugar a la enunciación de reglas abstractas y rigurosamente vinculadas entre sí que, una vez sistematizadas, pueden desvincularse por completo de la materia a la que se apliquen para constituirse en modelos de deducción aplicables a cualquier tema. La estructura formal de la deducción garantiza absolutamente la aceptabilidad de los teoremas a partir de la aceptación de los axiomas, con lo que, cumplida esta condición, se alcanza el mayor nivel de certeza entre los que pueblan nuestro sistema de pensamiento. A su vez, los axiomas se vuelven pragmáticamente aceptables cuando los teoremas que de ellos se derivan son útiles como estructuras para la construcción de modelos. Es posible trazar distintos sistemas deductivos en lógica, matemática o geometría, pero nuestra cultura se ha habituado a emplear predominantemente alguno de ellos y aun a considerarlo acríticamente aceptable, en virtud de la comprobación de su utilidad cotidiana. La aceptación efectiva de los sistemas deductivos más comunes es también muy alta, aunque no pueden descartarse errores o picardías en su aplicación, estas últimas más destinadas a su presentación ante terceros que al manejo personal del usuario. En este contexto, un fenómeno particularmente confuso es el que se observa en el discurso jurídico 
cotidiano: allí se encuentran a veces manifestaciones de menosprecio por las fórmulas matemáticas como algo ajeno o aun contrario a la dignidad humana, sin que sea fácil distinguir si se trata de casos en los que se procura sostener conclusiones que no derivan de premisas aceptables o, simplemente, de rechazo hacia lo desconocido.

\section{El contagio entre los niveles}

Entre los niveles de certeza apuntados, es posible trazar dos curvas. Una, correspondiente a la aceptabilidad, está en su punto más bajo en la preferencia y se eleva a tasa creciente hacia la deducción. La otra, que mide la aceptación individual de los criterios, encuentra en la preferencia su punto más alto, desciende hacia la moral y y vuelve a elevarse desde el derecho hacia la descripción y la deducción. En estas condiciones, no es extraño que estos dos últimos niveles, que reúnen aceptación y aceptabilidad (esto es, que tienden a ser común y sinceramente empleados), sean los más prestigiosos y se constituyan en paradigmas del conocimiento.

Las categorías de esos niveles (en especial la idea de verdad empírica o formal) tienden, pues, a extrapolarse hacia otros niveles en cuyos presupuestos no encajan adecuadamente. De ahí resulta cierta confusión en la que exigimos a unos niveles características de los demás, lo que genera diversas perplejidades.

Así, por ejemplo, la verdad (originaria del nivel 6 y objeto de abstracción en el nivel 7) es mencionada desde el nivel 2 como propiedad de ciertos principios y valores, y con mayor insistencia en el nivel 3 como aplicable a la descripción de ciertas normas éticas, lo que conduce a postular la existencia de hechos morales: en efecto, si la verdad es la condición de un enunciado que describe un hecho empírico, es necesario aceptar que hay hechos que hacen verdaderos los principios y las normas morales. En lugar de preguntarse si es apropiado aplicar a esos niveles aquella categoría, muchos dan por sentada dicha aplicabilidad y se lanzan afanosamente a buscar el lugar en que los hechos correspondientes puedan hallarse, así como el método que permita identificarlos.

El nivel 4, correspondiente a las normas jurídicas, es más propicio a los buceadores de hechos en los que pueda afincarse la verdad. Al menos ciertos hechos (sanción de las leyes, firma de los contratos, dictado de sentencias) se identifican intersubjetivamente como relevantes para la descripción del sistema jurídico y de las circunstancias de su aplicación. Pero este nivel sigue siendo un pariente pobre de los que le siguen, porque sus criterios, aunque bastante aceptables y relativamente aceptados, se hallan todavía muy lejos en estos aspectos de los criterios de la descripción empírica y de la deducción. Frente al derrumbe de una ciudad, a nadie se le ocurre plan- 
tear la nulidad del terremoto que la causó; ante una deducción cuyo resultado no nos satisface, no invocamos la inconstitucionalidad del modus ponens ni la incompetencia de quien haya demostrado el teorema.

El nivel 4, en el que nos movemos los juristas, es sometido así a exigencias de difícil cumplimiento. A menudo se le pide que dé cuenta de los hechos futuros (nivel 5), y que lo haga sin vulnerar su propia deducibilidad (nivel 7), lo que da lugar a las aporías de la cosa juzgada y del derecho consuetudinario. También se le reclama que incorpore las pretendidas verdades de los niveles 2 y 3, cuya impracticabilidad epistemológica constituye, precisamente, el fundamento pragmático del nivel 4: allí nos esperan la teoría iusnaturalista y el discurso acerca de la dignidad y de los derechos del hombre.

Para satisfacer tales exigencias, el derecho debería volverse proteico y ciertamente inconsistente: bajar su aceptabilidad hasta el nivel 2, elevarla hasta el nivel 7, introducir en sí mismo mutaciones extrasistemáticas con la esperanza de satisfacer el nivel 5, ajustar sus contenidos al nivel 3, presentarse como un segmento de la realidad al modo del nivel 6 y adquirir la solidez suficiente para resistir los embates del nivel 1.

¿Existe alguna esperanza de resolver estas perplejidades? En el estado actual del pensamiento normativo, la respuesta a esta pregunta parece claramente negativa. Pero, si quisiéramos a toda costa pensar positivamente, sería posible vislumbrar dos caminos: la integración y la segmentación.

La integración consiste en extender a los niveles inferiores, por lo menos desde el tercero, el grado de certeza en los criterios que es propia del nivel 6 o, por lo menos, del nivel 5. Ese objetivo requiere un consenso tan amplio en los niveles 3 y 4 como para constituir metacriterios ampliamente intersubjetivos para la solución de las controversias, lo que facilitaría en ellos el uso de la verdad y - quién sabe - también permitiría identificar algún segmento de la realidad como referente de sus enunciados verdaderos. El camino de la integración, como puede verse, es utópico: si la realidad social permitiera trazar semejante marco teórico, el derecho estaría desde hace siglos incluido en la moral y la teoría jurídica se reduciría al análisis de unos pocos instrumentos formales.

Elegir la segmentación, en cambio, consiste en trazar, al menos para los niveles 3 y 4, límites -también dotados de consenso- que proscriban en cada uno el uso de herramientas conceptuales concebidas para los otros; pero, además, asegurar a cada uno un lenguaje propio ajustado a su grado de certeza y de aceptabilidad, lenguaje que las personas puedan y quieran emplear para expresar su pertenencia individual al grupo que considera aceptables ciertos criterios y entenderse (es decir, comprender sus divergencias) con las personas que aceptan criterios distintos. Esta alternativa es un poco menos utópica, pero requiere un alto grado de conciencia en la construcción y 
en el uso de los lenguajes, que a su vez expresan presupuestos epistemológicos y hasta metafísicos.

Mientras tanto, una circunstancia podría ayudarnos a soportar nuestras perplejidades con menos sobresalto: la relativa coincidencia entre los contenidos del derecho y las preferencias de la mayoría, habitualmente agrupadas en cierto grado de consenso moral. Pero algunos piensan que ése sería el mayor de los milagros. El tiempo dirá. 